\title{
Experimental study on circular eccentric cam-follower pairs
}

\author{
Enrico Ciulli ${ }^{1)^{*}}$, Francesco Fazzolari ${ }^{2)}$, Bruno Piccigallo ${ }^{3)}$ \\ ${ }^{1)}$ Dipartimento di Ingegneria Civile e Industriale, University of Pisa, \\ Largo Lucio Lazzarino, I-56126 Pisa, Italy \\ 2) AMTesting s.r.l., Via di Torretta, S. Piero a Grado, 56125 Pisa, Italy \\ ${ }^{3)}$ Dipartimento Genio Navale, Accademia Navale - Livorno - Italy \\ "Corresponding author: ciulli@ing.unipi.it
}

\begin{abstract}
Experimental verifications of cam-follower contacts are very important because of the difficulties for a reliable simulation due to the continuous variation of load, speed and geometry of the lubricated contact. Some experiments have been carried out with a new apparatus, specifically designed and realised for investigation on cam-follower and gear teeth contacts, in order to test its capability to measure film thickness and contact forces. Circular eccentric cams have been used because they feature lower transient effects and comparison of the results with the theoretical/numerical ones is easier. The tests have been performed using cams with two different eccentricities and surface roughness, and two different followers, one made of steel and one made of glass.

The behaviour of the cam-follower contacts at several different rotational speeds, ranging from 50 to $500 \mathrm{rpm}$, and different pre-loads have been investigated. Lubrication regimes ranged from boundary to complete, being most of the tests performed under mixed lubrication conditions. Localised wear has occurred during some tests. Data of all contact force and moment components as well as of the cam shaft driving torque have been acquired at high acquisition frequency. Some methodologies for numerical data elaboration have been identified. Optical interference images have been correctly recorded at the desired frequency. The trends of the normal and friction forces measured in the different tests are presented in the paper, as well as the evaluated trends of the friction coefficient. Some sample optical interference images are also shown. The results furnish encouraging indications about the capabilities of the experimental apparatus.
\end{abstract}

Keywords: cam, follower, lubricated contacts, friction, film thickness, experimental rig

\section{Introduction}

Cam-follower pairs are difficult to simulate because of the continuously variable conditions of load, speed and geometry. As reported in [1], some experimental rigs can be found in literature that have been realised and used for measuring friction and film thickness, with different techniques, in this kind of contact.

Some friction measurements are reported for instance in [2]. The total friction torque is measured by a strain gauge torque transducer. The power loss associated with the cam-follower contact is evaluated by subtracting the the other losses of the apparatus. A direct measurement of the contact force components is performed in [3] by using a system with three load cells. Friction and wear measurements for investigating the influence of lubricant additives and surface coatings, finish and texture are performed in [4], [5], [6], [7]. Tests on real engines have been also performed, as for instance in [8].

Attempts of direct measurements of oil film thickness are reported in [9] where a capacitance transducer is used, in [10] with thin film micro transducers, in [11] with electrical resistivity and in [12] with optical interferometry. Optical interferometry is one of the most detailed method for measuring film thickness and shape, also when a continuous variation of the working conditions during running occurs, as for a cam-follower contact. Investigations on nonconformal lubricated contacts under transient conditions are reported for instance in [13], [14], [15].

A new test rig has been designed and realized for investigations on contact force and lubricant film thickness and shape in cam-follower contacts [16]. In this work, some experimental results obtained in tests carried out for testing the capabilities of the apparatus for measuring forces and film thickness are presented.

\section{Test rig}

The experimental apparatus, that reproduces the cam follower contact, is shown in Figure 1. It has been specifically designed and realised for investigation on cam-follower and gear teeth contacts. It is able to simultaneously measure all the contact-force components as well as film thickness and shape. Optical interferometry is used for the latter. 

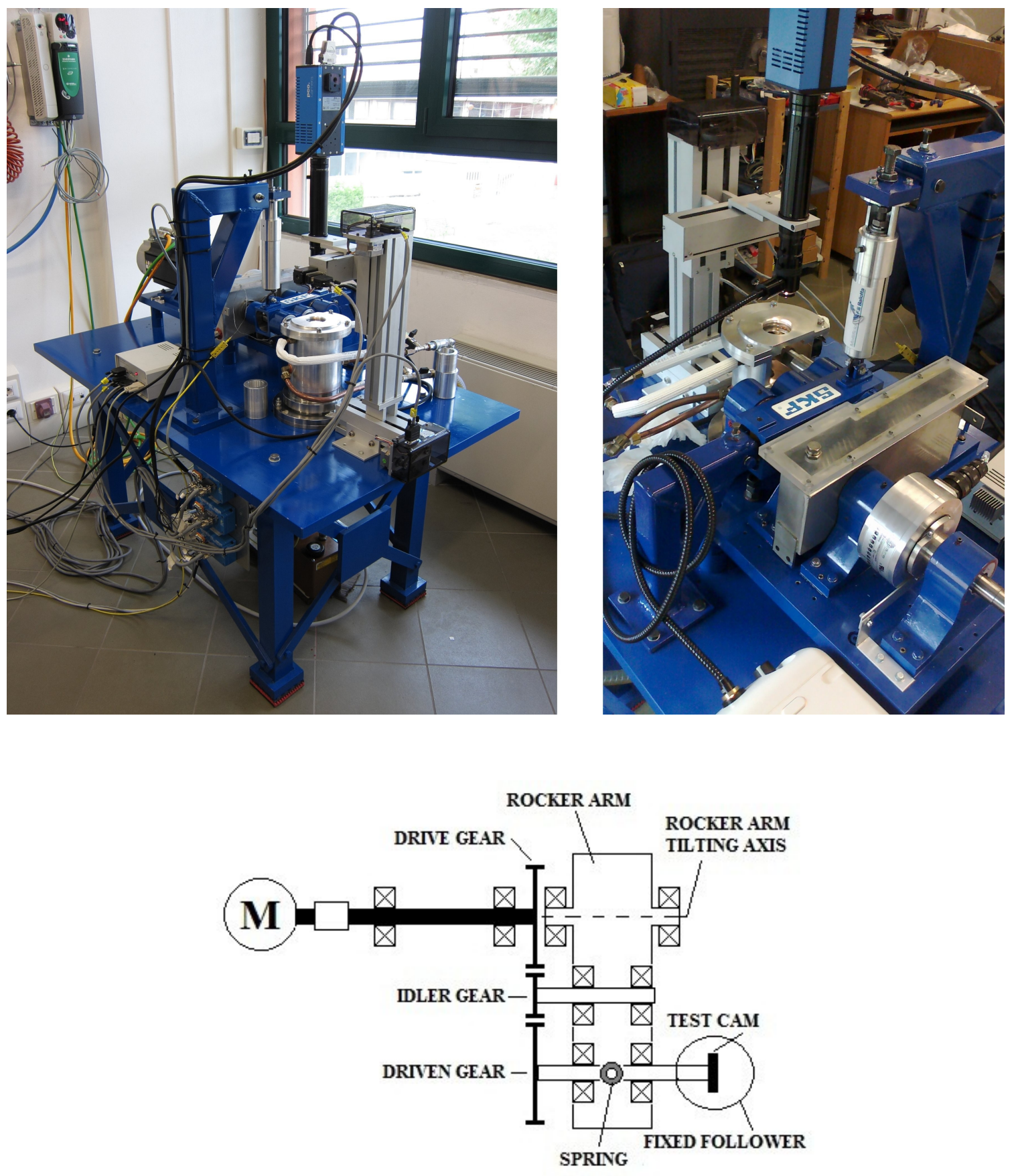

Figure 1 Photographs (a) showing a global view (left) and a detail of the power transmission and measuring zone (right) and schematic drawing (b) of the test rig.

Two different configurations are possible: with fixed or moving follower. The follower position is maintained steady when optical interferometry has to be performed (this is the case of this study), meanwhile the cam can simultaneously rotate and move up and down being coupled with a shaft borne on a rocker arm.

The cam is driven by an electrical brushless motor through an epicyclic gear system with gear ratio equal to 1 . The rocker arm can tilt around a fixed axis coaxial with the one of the driving gear. Driven and idler gears are mounted on two shafts located on the rocker arm by tapered bearings. The cam under test is mounted on the driven gear's shaft. The contact between cam and follower is granted by an interchangeable spring. The cam remains in contact with a fixed tappet, thus the tilting motion of the rocker arm depends on the cam's profile.

Thanks to the use of an adaptor, the follower can be easily changed in order to test different types of materials and surface coatings or using glass discs with semi-reflective coatings in order to perform optical interferometry. The adaptor is retained in a novel six axes dynamometer, based on piezoelectric sensors, specifically designed and manufactured for the test rig [2,3]. The dynamometer is used for the measurement of all contact force components, along three directions (normal to the follower, parallel to the cam axis and a third direction orthogonal to the previous 
two); its calibration was carried on by a specific equipment, applying known pure forces and torques by dead weights as reported in [16], [17].

The lubricant is directly furnished to the contact area by a low-pressure oil system and it is maintained to the required temperature by a thermostatic bath. The lubricant temperature is measured at the inlet by the means of a $\mathrm{K}$ thermocouple.

The data acquisition system, developed on a National Instruments ${ }^{\circledR}$ C-Rio platform, permits the simultaneous and synchronous sampling of contact force and angular position of the cam. All the data are usually sampled at rate $10 \mathrm{kHz}$ for each channel with a resolution of 24 bits.

A high-speed camera coupled to a microscope is used for recording the interference images from which the film thickness can be evaluated. The optical system is mounted on a computer-controlled XYZ positioning device so that it is able to follow the contact area during the cam's rotation. A program has been developed in Labview ${ }^{\circledR}$ for the movements along the three axes.

Some additional technical data of the test rig are reported in Table 1.

Table 1 - Technical data

\begin{tabular}{lc}
\hline Maximum speed (rpm) & 3000 \\
Motor power (kW) & 5.65 \\
Maximum measurable normal force (N) & 6000 \\
Maximum measurable friction force (N) & 900 \\
Maximum cam's lift (mm) & 20 \\
\hline
\end{tabular}

\section{Experimental details}

Some experiments have been planned in order to set up the experimental procedures, to verify the capabilities of the apparatus, to develop a data processing methodology and to detect possible problems. Circular eccentric cams with spherical surfaces have been tested against the plane surface of discs simulating the follower. The circular eccentric cams have been chosen because they give rise to lower inertia forces and transient effects compared to the real engine cams, as well as for the ease of obtaining kinematic and dynamic data from analytical models.

The contact zone is shown in Figure 2. In Figure $2 b$ the used coordinate system and the notation of the contact force components are reported.

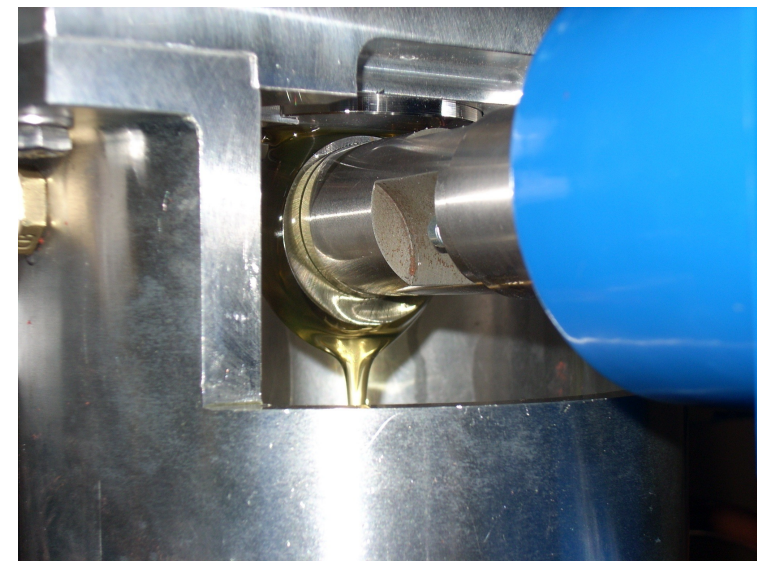

a

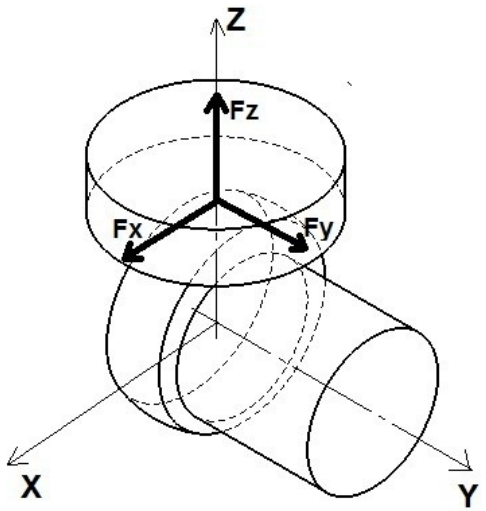

b

Figure 2 Photograph (a) and schematic drawing (b) of the cam-follower contact.

For a better understanding of the experimental results reported in the following, it is worth reminding here briefly the essential geometry and kinematic aspects of the investigated contact.

Being $r$ and $e$ the cam radius and eccentricity respectively, with reference to the coordinate system in Figure 3 it is easy to obtain the position and the velocity of the contact point $P$ :

$$
x_{P}=-e \sin (\theta) \quad, \quad v=-e \omega \cos (\theta)
$$

where $\theta$ is the cam rotation angle with $\theta=0^{\circ}$ corresponding to the minimum distance between the cam axis and the contact point, when the contact is on the base circle of radius $r-e$. 


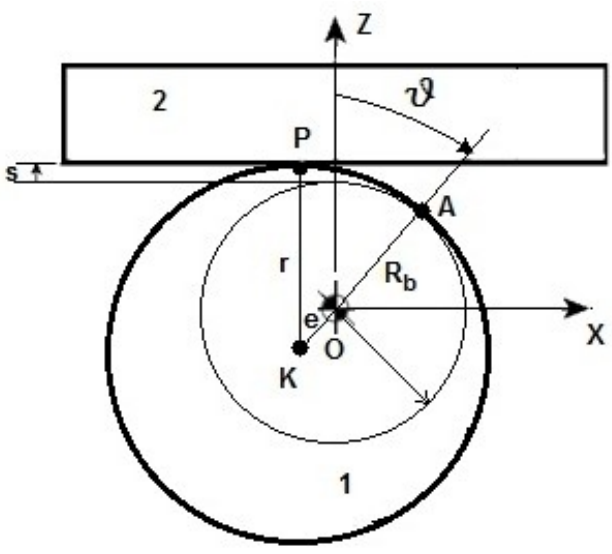

Figure 3 Geometric quantities of the cam-follower contact.

The physical points of the two bodies in contact in $P$ have the same velocity in the $z$ direction but different ones in the $x$ direction. The $x$ component of the follower velocity is zero while the $x$ component of the cam's point velocity is $v_{x}=z_{P} \omega$, where $\omega=\dot{\boldsymbol{\theta}}$ is the angular velocity and $z_{P}$ the follower position:

$$
z_{P}=r-e \cos (\theta)
$$

Therefore, the entraining velocity $u$ can be calculated as:

$$
u=\frac{\left(v_{x}-v\right)+(0-v)}{2}=\frac{r+e \cos (\theta)}{2} \omega
$$

Sample contact point and entraining velocities are shown in Figure 4, as well as the follower lift:

$$
s=e[1-\cos (\theta)]
$$

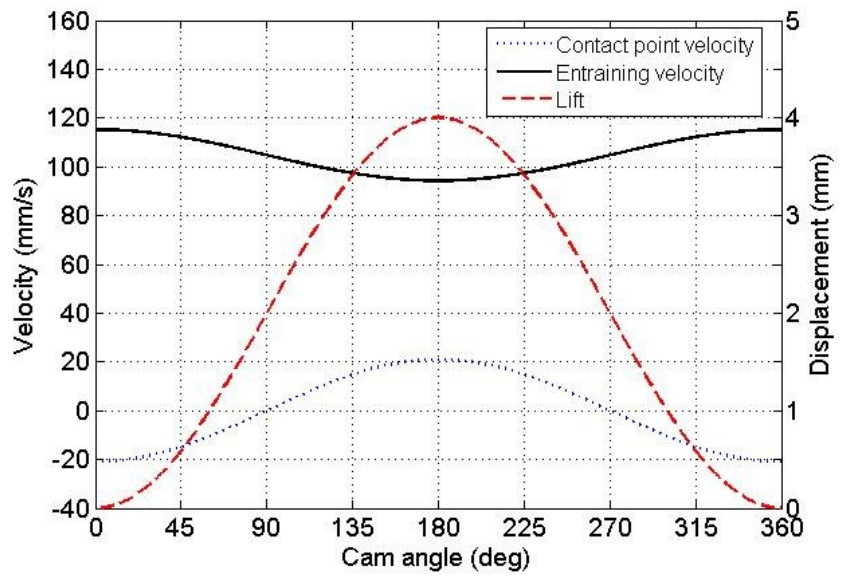

Figure 4 Contact point velocity, entraining velocity and follower lift evaluated for a circular cam with eccentricity $=2 \mathrm{~mm}$ at $100 \mathrm{rpm}$.

It is worth noting that the lift usually corresponds to the movement of the follower while it corresponds to the movement of the cam axis in the investigated conditions. It is evident that the contact point velocity changes its direction every $180^{\circ}$ while the entraining velocity has always the same direction.

The cams used for the tests reported in this paper are made of C40 steel and have a radius of curvature of $20 \mathrm{~mm}$. Cams with different roughness have been used with a root mean square roughness Rq ranging from about 0.1 to $1 \mu \mathrm{m}$. Two different eccentricities have been tested, $e=2 \mathrm{~mm}$ and $e=4 \mathrm{~mm}$. In Figure 5 a picture of a cam is shown. 


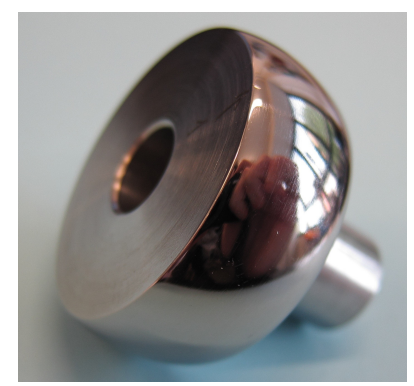

Figure 5 Picture of a cam used during tests.

The cams have been tested against the plane surfaces of two kinds of followers, one made of steel with $\mathrm{Rq} \approx 1.3 \mu \mathrm{m}$ used for tests where only forces are measured and one made of glass with $\mathrm{Rq} \approx 0.03 \mu \mathrm{m}$ for measuring film thickness too. Actually the roughness data are reported only as a rough indication, because of the presence of local significant roughness variations due to wear occurred at the beginning of some tests particularly on the followers surfaces and due to the manual lapping used for obtaining the low roughness spherical surfaces. The manual lapping created also problems of roundness evidenced by the interference images shown later in the paper. However, these aspects were not significant for the main purpose of this work.

Three groups of tests were planned.

The first group of tests was addressed to the evaluation of the contact forces only. The cam with the highest roughness and eccentricity $2 \mathrm{~mm}$ has been tested against the steel follower.

The second group of tests has been performed with a low roughness spherical cam with $e=2 \mathrm{~mm}$ and a glass disc with a semi-reflecting coating ( $\mathrm{Cr}$ layer $145 \AA$ thick plus protection $\mathrm{SiO}_{2}$ layer with $600 \AA$ thickness) for the interferometric measurements.

The third group of tests has been performed with the same follower and a cam similar in roughness to the one employed in the second group, but with a greater eccentricity $e=4 \mathrm{~mm}$.

A spring with a stiffness of $20.22 \mathrm{~N} / \mathrm{mm}$ has been employed. Different pre-loads of 83, 89 and $10 \mathrm{~N}$ have been used for the first, second and third group of tests respectively. The pre-load was applied with the contact point on the cam base circle $\left(\theta=0^{\circ}\right)$.

The same oil was used in all tests, a standard motor oil SAE 5W-40 continuously filtered and refilled with a contact inlet temperature of $26 \pm 1{ }^{\circ} \mathrm{C}$. The lubricant viscosity at test temperature was about 0.14 Pas.

Seven different velocities have been used for the first group of tests: 50, 100, 200, 250, 300, 350 and 400 rpm; two for the second group: 400 and $500 \mathrm{rpm}$; and three for the third one: 100, 300 and $500 \mathrm{rpm}$.

\section{Data acquisition and processing}

For each test at a different cam rotation regime several data are acquired, including all components of the contact force and the torque applied to the cam shaft. About 5 cam rotation cycles are sampled for each condition in order to have a significant amount of data but a short duration of each test, so that the drift effect introduced by the charge amplifier of piezoelectric sensors can be ignored.

Data are recorded with cam angular resolutions ranging from $0.03^{\circ}$ at $50 \mathrm{rpm}$ up to $0.3^{\circ}$ at the maximum velocity reached, $500 \mathrm{rpm}$. All data are saved in a hard disc for post processing.

The voltage signals acquired from the six-axes dynamometer are converted in real time into the force and torque components in the acquisition program, developed in Labview ${ }^{\circledR}$, by means of a calibration matrix. The relevant data can hence be visualized during the test with no delay, thanks to the simultaneous sampling capacity of the dAQ channels.

The data are stored either unscaled (in volt) or scaled (force and torque). Some data manipulation is performed on the acquired data using a number of Matlab ${ }^{\circledR}$ scripts. Besides the signal scaling, using the calibration matrix, they realise operations like spectral analysis, filtering and plotting.

In Figure 6 an example of unfiltered and filtered signals is shown. 


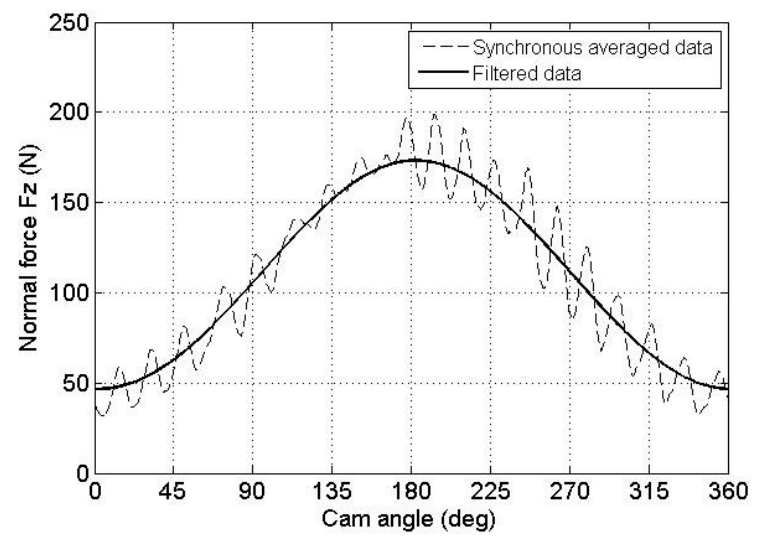

Figure 6 Sample unfiltered and filtered data. Contact normal force in the third group of tests at $300 \mathrm{rpm}$.

The noise affecting the signal, which amplitude increases with the cam's speed, is mainly due to the transmission realised by an epicyclical gear system with helical gears (input and output gears with 40 teeth, idle gear with 16 teeth).

A numerical low pass $5^{\text {th }}$ order Butterworth filter was implemented in Matlab ${ }^{\circledR}$, using different cut-off frequencies, in order to obtain filtered signal for the contact force/torque.

\section{Experimental results}

Force measurements have been made in all tests. Interference images have been recorded only in the second and third group of tests.

\subsection{Force and torque measurements}

The normal contact force $F_{z}$ measured in a cam cycle for some different rotation speeds of the first group of tests (cam eccentricity $2 \mathrm{~mm}$, steel follower) is shown in Figure 7. The results obtained with the simulation model described in [18] are also shown in the same figure (only data measured every $10^{\circ}$ have been reported on the diagram for making the lines representing the calculated values distinguishable).

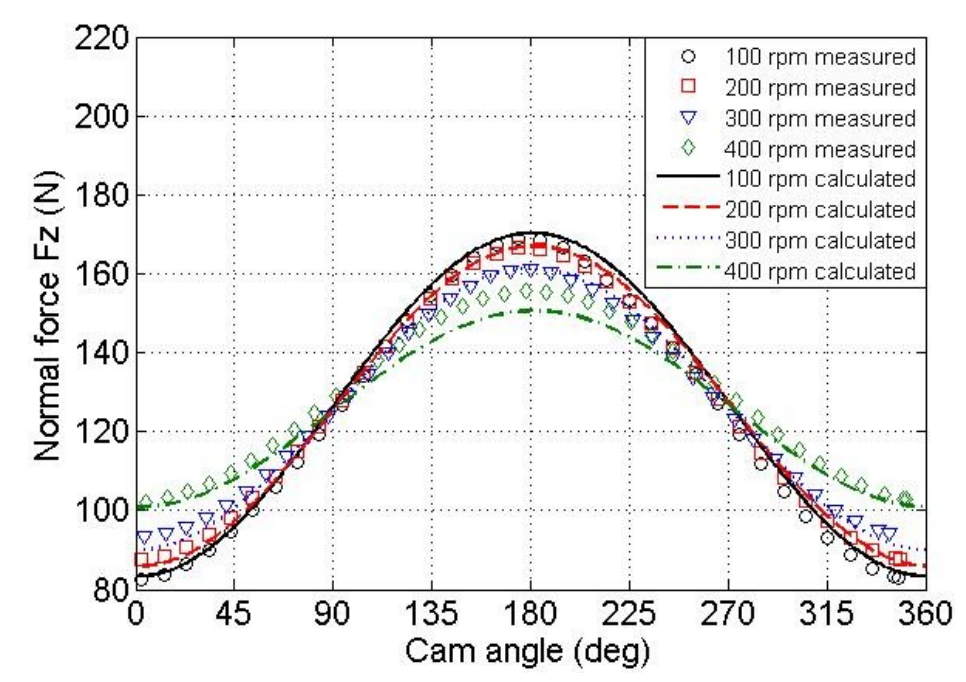

Figure 7 Measured and calculated normal contact force $F_{z}$ versus cam rotation angle for several speeds (first test group).

The total amount of the normal contact force is due to the superposition of the elastic and inertia forces [18].

The maximum value of the elastic force, depending on the restoring spring, is in correspondence to the cam nose $\left(180^{\circ}\right)$. The dynamic effect, depending on the acceleration, has also its maximum amplitude by the cam nose, but in opposition of phase with respect the elastic force. In fact, it is easy to verify by derivation of eq. (2) that, being $\omega$ constant, the follower acceleration is: 


$$
\ddot{z}_{P}=e \omega^{2} \cos (\theta)
$$

which corresponds to a negative inertia force that increases the normal contact force between 0 and $90^{\circ}$ and between $270^{\circ}$ and $360^{\circ}$ and a positive one that decreases the load between 90 and $270^{\circ}$.

The increasing effect of the inertia force by increasing the rotational speed of the cam is evident in Figure 7: the maximum of $F_{z}$ passes from $170 \mathrm{~N}$ at $100 \mathrm{rpm}$ to $155 \mathrm{~N}$ at $400 \mathrm{rpm}$ in fairly good accordance with the calculated results.

The corresponding friction force $F_{x}$, Figure 8, decreases with the increase of the cam rotation speed, along all cam rotation angles. It can be noticed the angular displacement of the maximum value of the $F_{x}$ as the rotation speed is increased. It goes from about $170^{\circ}$ (close to the cam's nose) at $50 \mathrm{rpm}$, to $130^{\circ}$ at $400 \mathrm{rpm}$ (Figure 9).

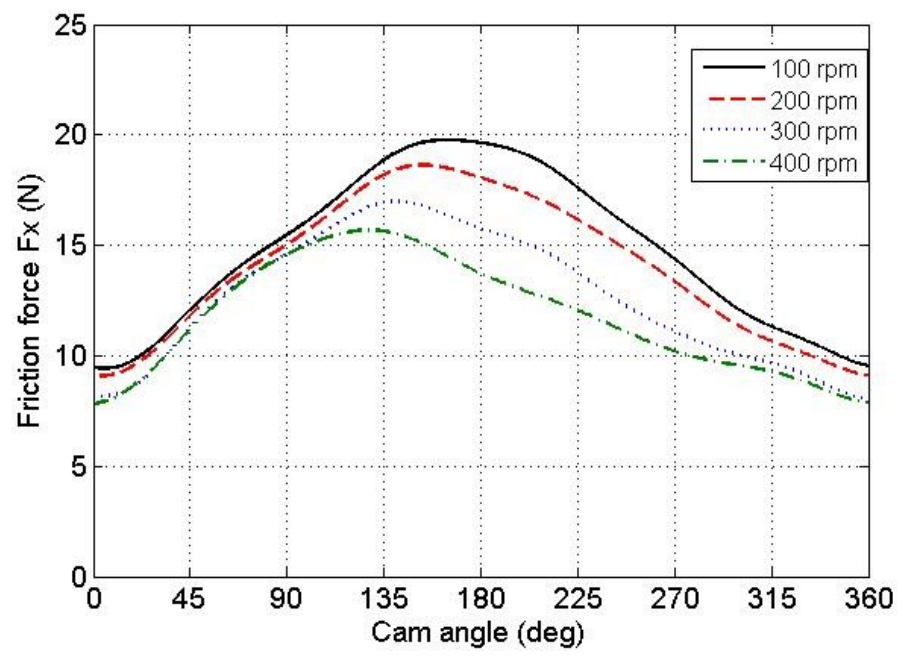

Figure 8 Friction force $F_{x}$ versus cam rotation angle measured in the first group of tests for several speeds.

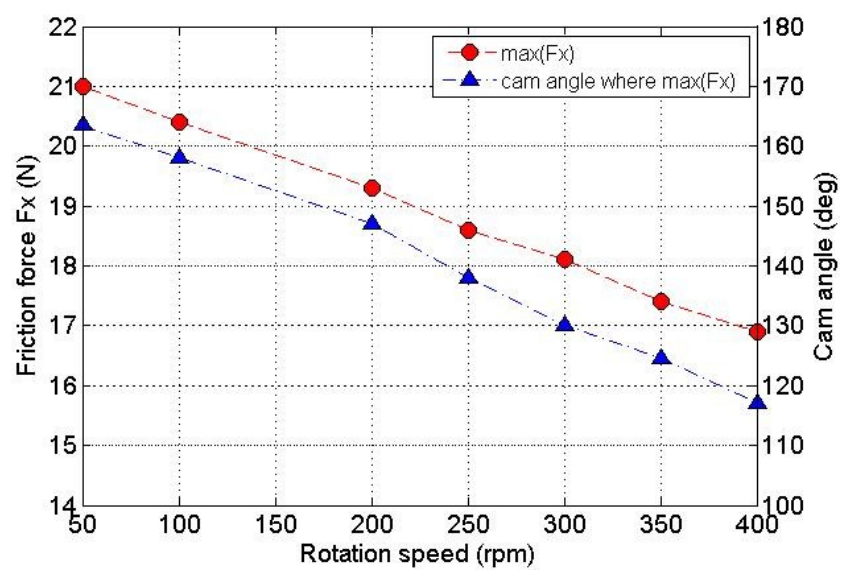

Figure 9 Friction force maximum value (red circle) and its angular position (blue triangle) versus cam rotation speed in the first group of tests.

The same trends of the contact normal force have been also recorded in the second group of tests. 
Regarding the friction force, the results for $400 \mathrm{rpm}$ are shown in Figure 10. The friction force is smaller than the one measured in the first group of tests because of the lower roughness of surfaces in contact. The displacement of the maximum value also occurs in this test. The results at $500 \mathrm{rpm}$ are not reported because they are not significant due to wear problems on the cam and on the glass disc, as evident by the interference images reported later in the paper.

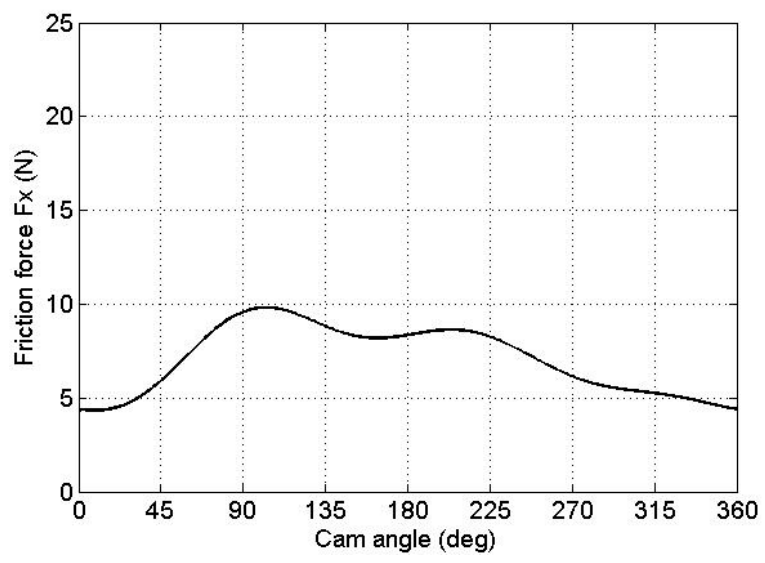

Figure 10 Friction force $F_{x}$ versus cam rotation angle measured in the second group of tests at $400 \mathrm{rpm}$.

In order to avoid the wear problems encountered in the second group of tests due to the high pre-load, some additional tests have been made with a lower pre-load in the third group of tests. The normal contact force recorded is shown in Figure 11. The inertia effects are more evident than in the previous tests because of the greater eccentricity of the cam (4 mm instead of $2 \mathrm{~mm}$ ). Similarly to Fig. 7, calculated values are also reported in the diagram.

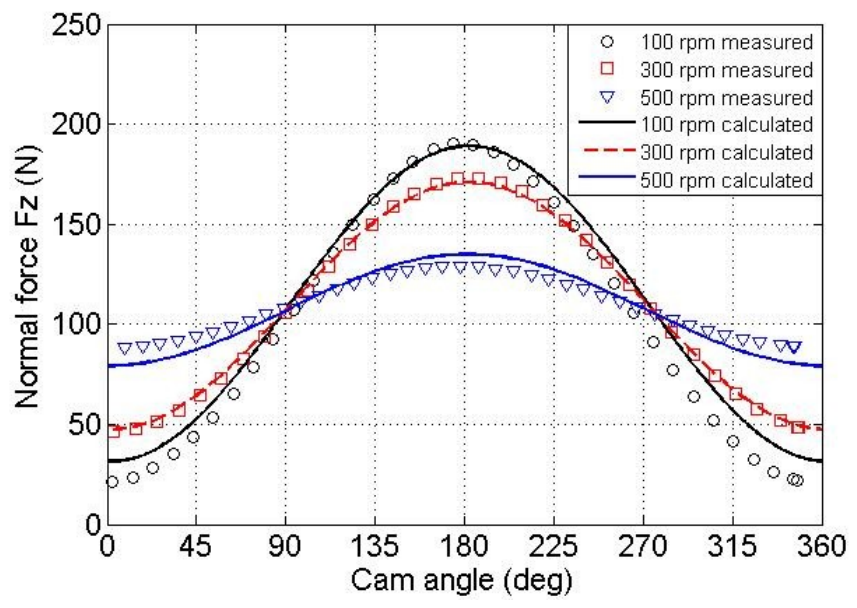

Figure 11 Measured and calculated normal contact force $F_{z}$ versus cam rotation angle for several speeds (third test group).

The measured friction force is shown in Figure 12. 


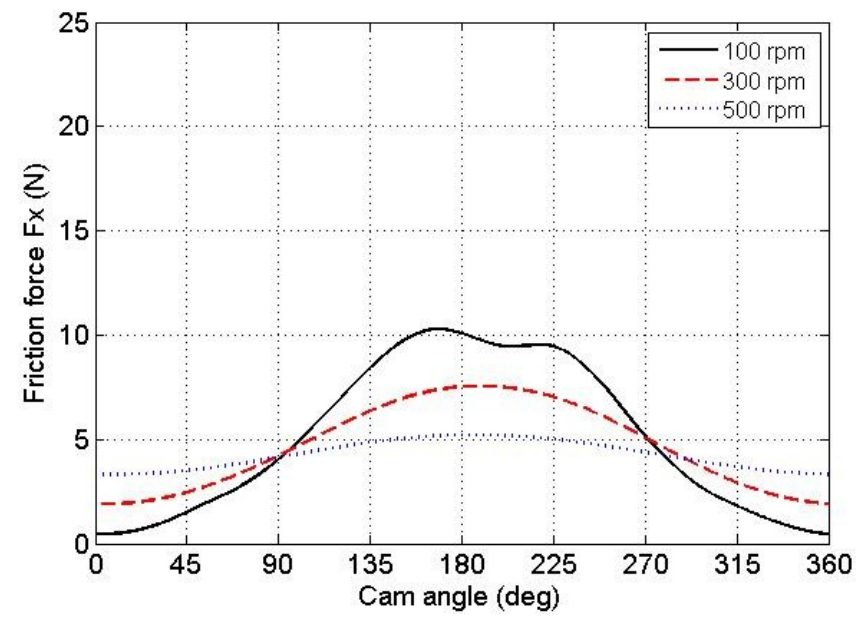

Figure 12 Friction force $F_{x}$ versus cam rotation angle measured for several speeds (third test group).

An example of cam driving torque, directly measured thanks to the dynamometer, is shown in Figure 13 . The negative offset is due to the friction force, while the main part of the moment is due to the normal force. The torque takes negative values for the whole up side of the lift curve, then it changes its sign at approximately $215^{\circ}\left(35^{\circ}\right.$ after the cam nose, where the maximum lift occurs), and it becomes negative again at $325^{\circ}$.

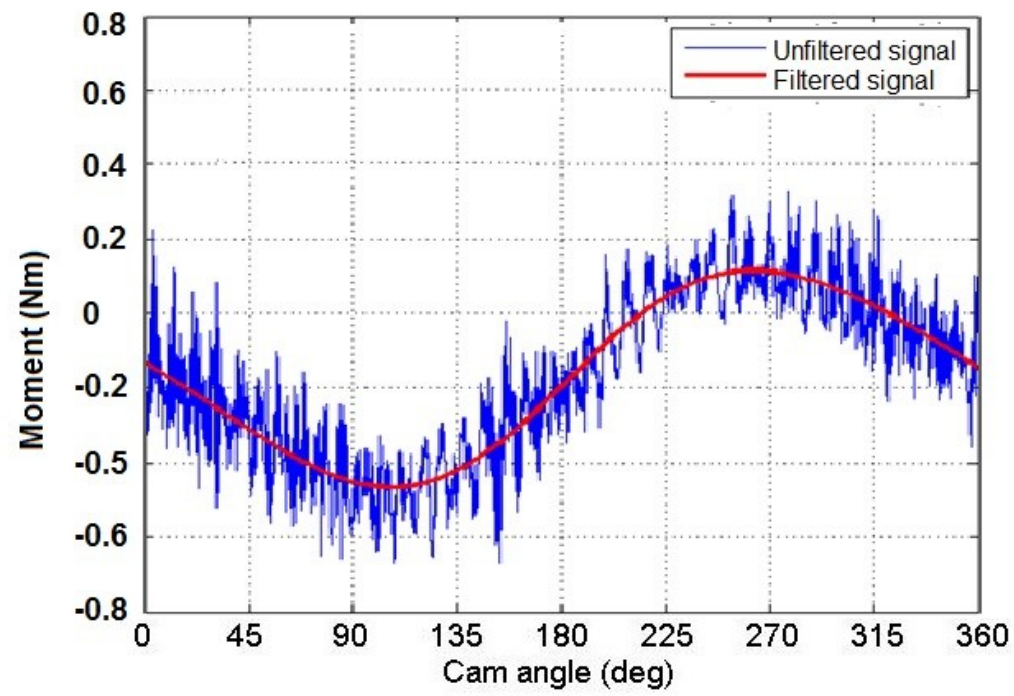

Figure 13 Unfiltered and filtered cam driving torque trends at $300 \mathrm{rpm}$ (first test group).

During the negative torque phases, the cam follower tends to brake the motor, while during the positive phase it gives back work into the drive system. The change of sign is due to the contribution of the normal contact force that in the half part of the cam cycle has a positive moment; its lever is maximum $(2 \mathrm{~mm})$ at the 270 degree position (where the motion of the cam follower contact point inverts its direction).

\subsection{Interference images}

For all tests the microscope with the camera was not moved. The magnification was adjusted in order to include the entire path of the contact point. The images were recorded at $1200 \mathrm{fps}$.

In Figure 14 some interference images recorded at $400 \mathrm{rpm}$ in the second group of tests are shown. Some problems due to scratches on the semi-reflecting layer provoked by the pre-load applied to the contact before the starting of the cam rotation are visible at $0^{\circ}$ and $90^{\circ}$. 


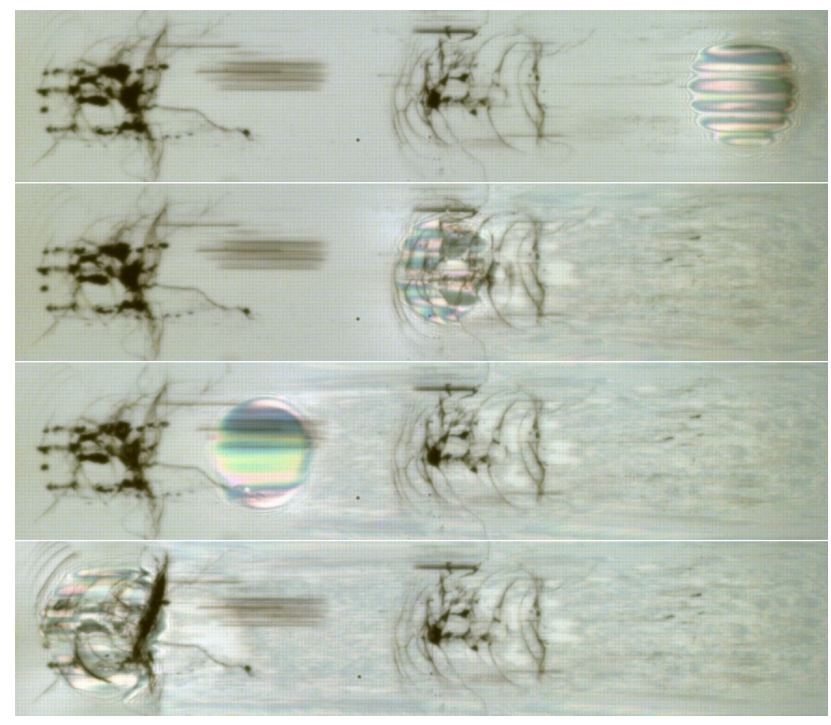

Figure 14 Examples of interference images recorded at 400 rpm in the second group of tests. From the top: $-90^{\circ}, 0^{\circ}$, $30^{\circ}, 90^{\circ}$ of cam rotation angle. Dimension of each image: $5 \times 1.1 \mathrm{~mm}$.

Another problem is connected with the non uniform roughness of the cam surface, that clearly shows grinding marks at certain angular positions.

Some interference images recorded for three velocities in the third group of tests are shown in Figure 15, 16 and 17. In all figures, the pictures with the interference image located in the centre corresponds to $0^{\circ}$ of cam rotation angle when the load is lower and $180^{\circ}$ when it is higher (the load intensity is evidenced by the dimension of the interference image). The pictures with the interference image on the left corresponds to $90^{\circ}$ and on the right to $270^{\circ}\left(-90^{\circ}\right)$. The contact point movement is from right to left $\left(-90^{\circ}, 0^{\circ}, 90^{\circ}\right)$ then from left to right $\left(90^{\circ}, 180^{\circ}, 270^{\circ}\right)$. The entraining velocity is always from left to right.

The contact appears to be mainly under mixed lubrication conditions with possible direct localised solid-solid contacts particularly related to the presence of the machining marks. The roughness and shape lack of homogeneity of the cam are also evident. Complete lubrication conditions could occur only at the highest speed around $0^{\circ}$ of cam rotation angle, where the entraining velocity is maximum and the load minimum.

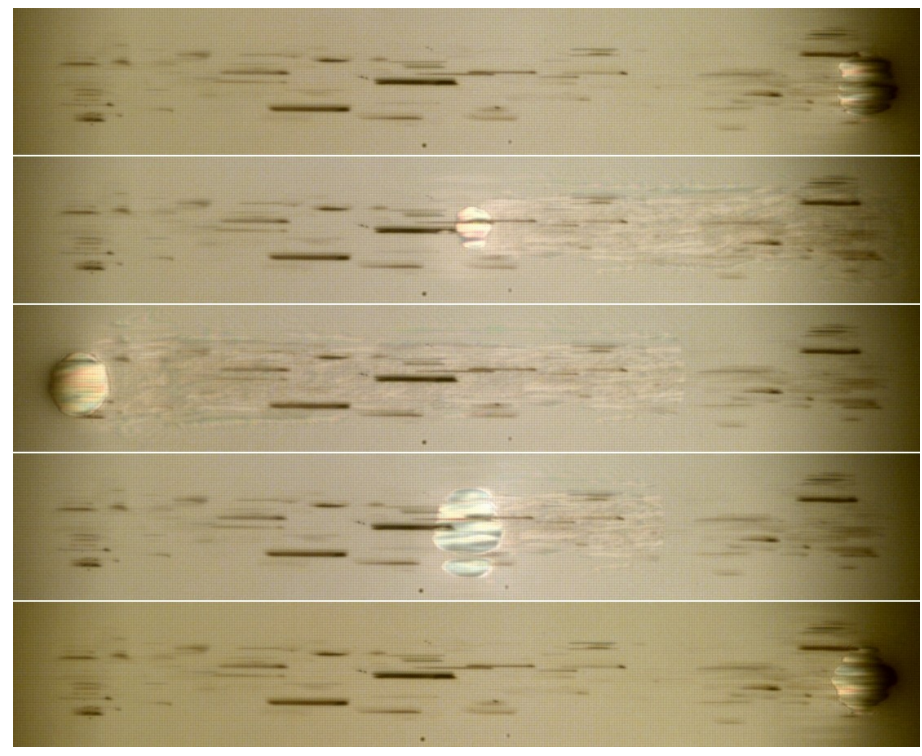

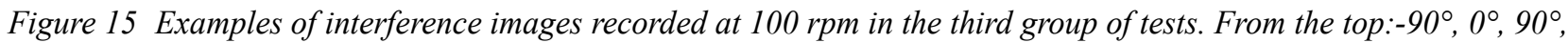
$180^{\circ}$ and $270^{\circ}$ of cam rotation angle. Dimension of each image: $9.3 \times 1.5 \mathrm{~mm}$. 


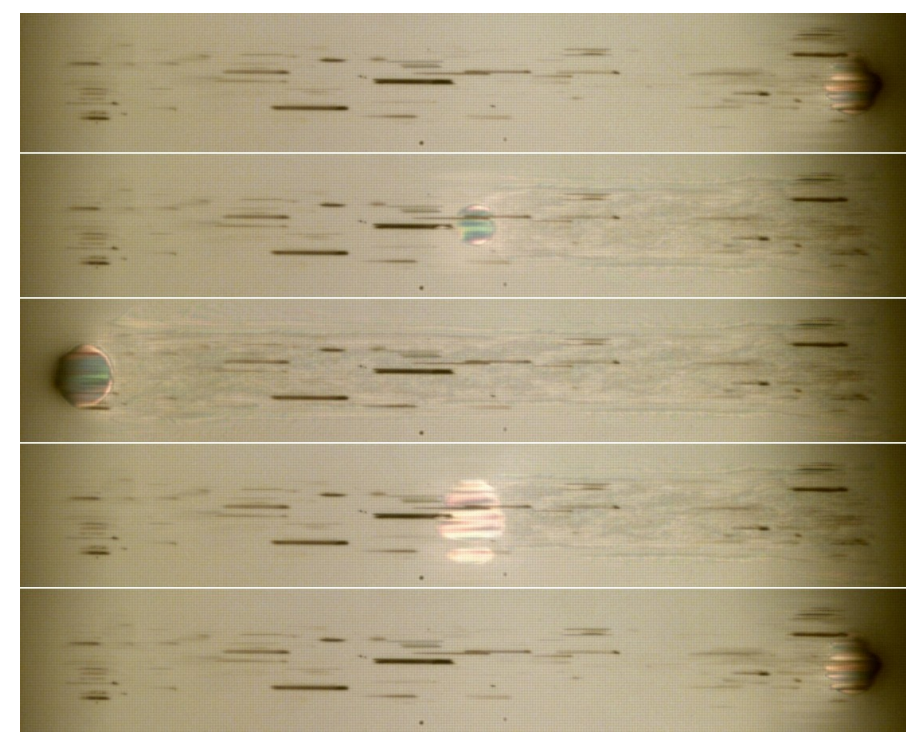

Figure 16 Examples of interference images recorded at $300 \mathrm{rpm}$ in the third group of tests. From the top:-90 $90^{\circ}, 90^{\circ}$, $180^{\circ}$ and $270^{\circ}$ of cam rotation angle. Dimension of each image: $9.3 \times 1.5 \mathrm{~mm}$.

Figure 17

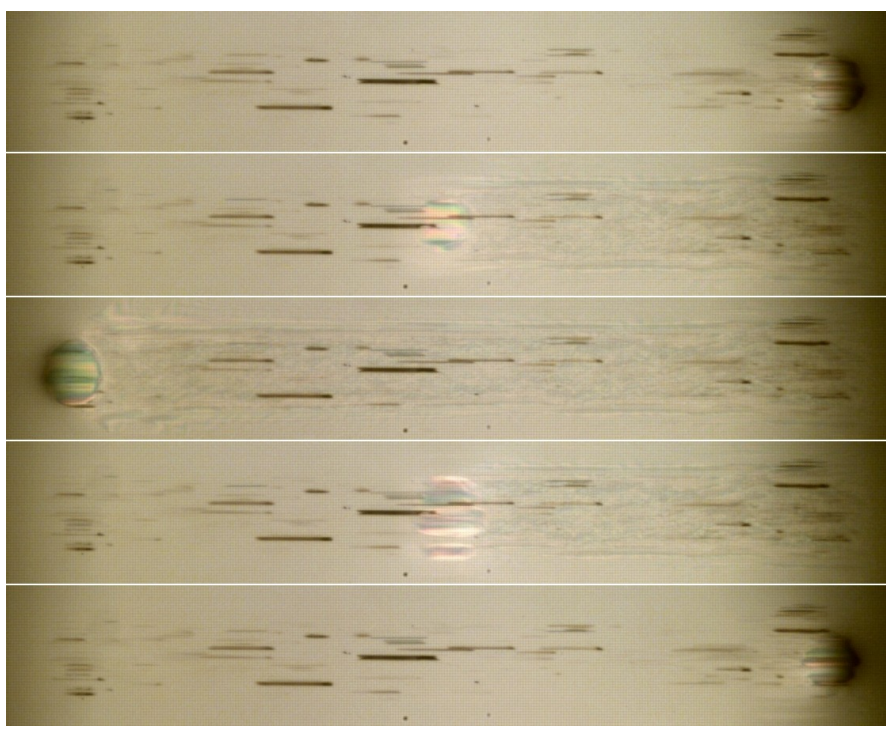

Examples of interference images recorded at $500 \mathrm{rpm}$ in the third group of tests. From the top:-90 $, 0^{\circ}, 90^{\circ}, 180^{\circ}$ and $270^{\circ}$ of cam rotation angle. Dimension of each image: $9.3 \times 1.5 \mathrm{~mm}$.

For deeper comparisons, six successive recorded images are shown in each column of Figure 18. Images of the first three columns have been recorded during the test at $300 \mathrm{rpm}$ in correspondence of three different cam angular positions: close to $0^{\circ}, 90^{\circ}$ and $180^{\circ}$. The last two columns show images recorded close to $180^{\circ}$ at 100 and $500 \mathrm{rpm}$.

By comparing images of first three columns, the different velocities of the contact point are evident (the absolute value of the contact point velocity is maximum at $0^{\circ}$ and $180^{\circ}$ and minimum at $90^{\circ}$, according to eq. (1) and Figure 4).

The lower film thickness at $100 \mathrm{rpm}$ is evident by comparing images taken around $180^{\circ}$ for the different cam rotational speeds. The dark zones in the interference images recorded at $100 \mathrm{rpm}$ evidence direct contact of the bodies.

The specimen appears not perfectly spherical particularly at $180^{\circ}$ where it has been worked again because some wear occurred in some previous tests. 


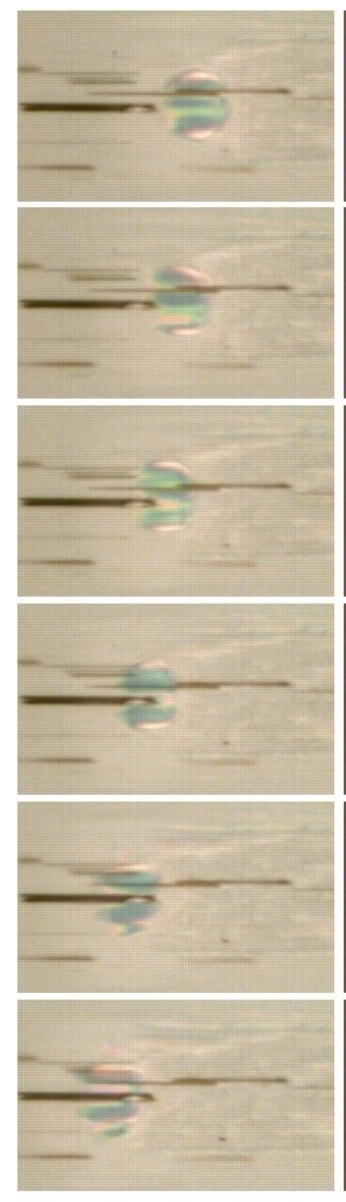

$300 \mathrm{rpm}-0^{\circ}$
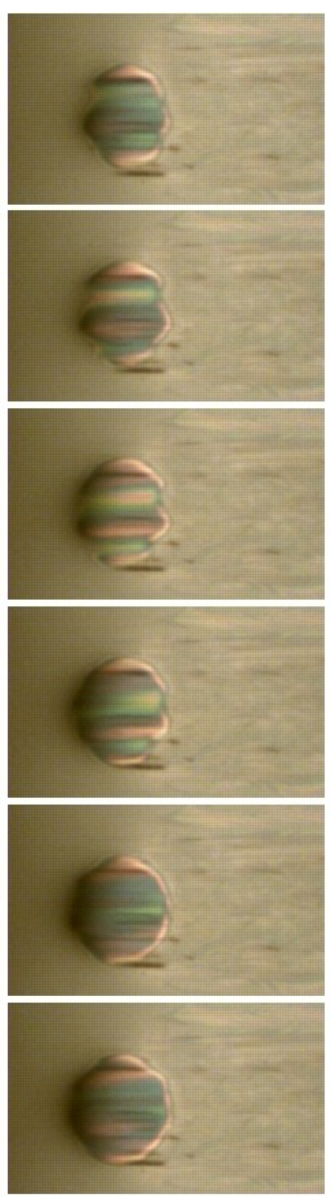

$300 \mathrm{rpm}-90^{\circ}$
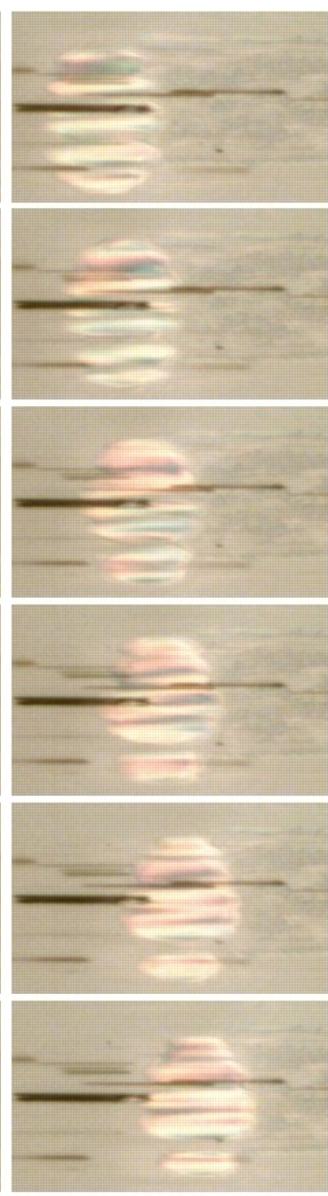

$300 \mathrm{rpm}-180^{\circ}$
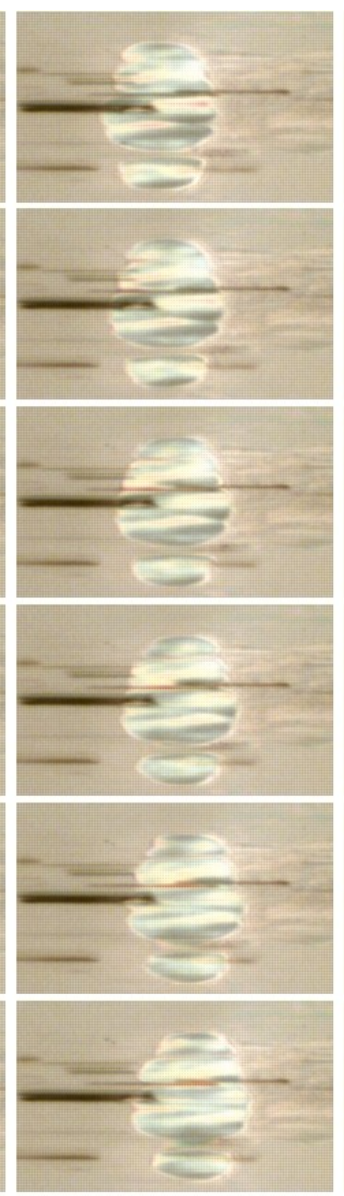

$100 \mathrm{rpm}-180^{\circ}$
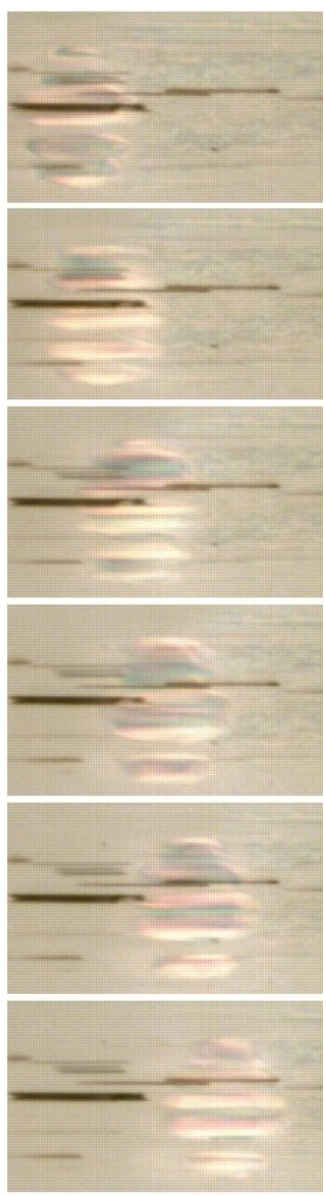

$500 \mathrm{rpm}-180^{\circ}$

Figure 18 Details of interference images recorded at different rotational speeds and in the central position with the minimum $\left(0^{\circ}\right)$ and maximum $\left(180^{\circ}\right)$ load and at the maximum contact point displacement $\left(90^{\circ}\right)$. In each column six successive images recorded every $0.83 \mathrm{~ms}$. Dimension of each image: $1.9 \times 1.2 \mathrm{~mm}$.

\section{Discussion}

The analysis of the interference images jointly with the trends of the friction coefficient furnishes useful indications for understanding what occurred during the tests carried out.

The friction coefficient is calculated as the ratio between the friction force amplitude measured in $x$ direction and the normal contact force amplitude measured in $z$ direction.

The friction coefficient trends for the first group of tests are plotted in Figure 19. The diagram shows a decrement as speed is increased and an asymmetry with respect the cam's nose. The highest value is near the first inversion of the motion of the contact point, during the up side of the lift curve, and this effect become more evident as speed is increased.

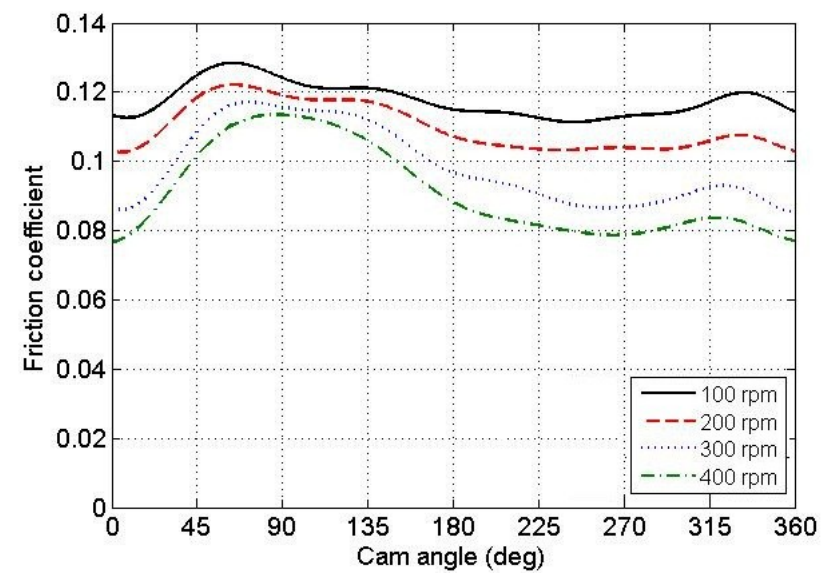

Figure 19 Friction coefficient versus cam rotation angle in the first group of tests for four speed values. 
The values of the friction coefficient clearly indicate boundary lubrication condition for most part of the contact. Mixed conditions are likely to occur for the highest values of the cam rotational speed in the positions where the entraining velocity takes its maximum value. It is noteworthy that the cam presented a zone with greater wear around $90^{\circ}$.

The friction coefficient calculated at $400 \mathrm{rpm}$ for the second group of tests is shown in Figure 20 and for the third group in Figure 21.

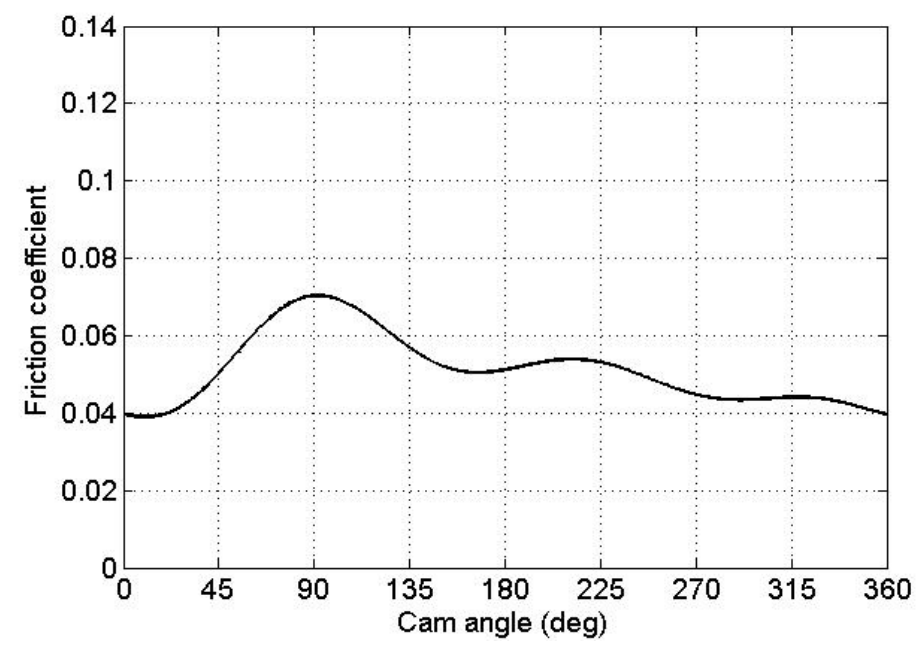

Figure 20 Friction coefficient versus cam rotation angle at $400 \mathrm{rpm}$ in the second group of tests.

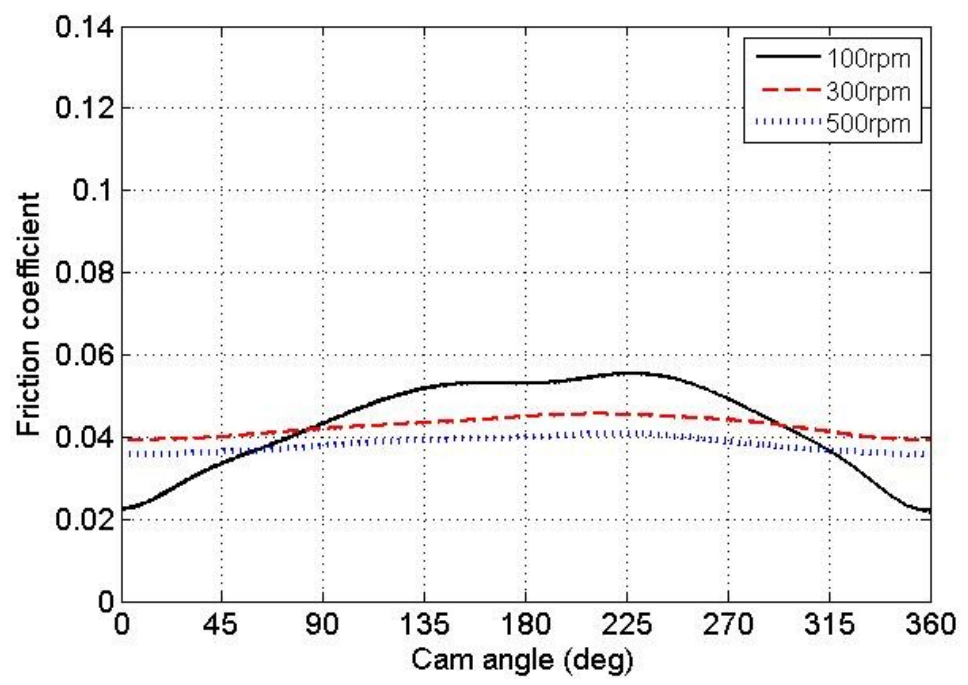

Figure 21 Friction coefficient versus cam rotation angle in the third group of tests for three speed values.

The values of the friction coefficient are lower compared to the previous ones, showing a prevalent mixed lubrication condition.

The peak on the friction coefficient plot in Figure 20 is related to the worn zone of the cam (the same used for the test of first group).

The friction trends in Figure 21 indicate that, due to the lower roughness of the follower in the new contact point (absence of the cracks present in Figure 14) and to the absence of a worn zone in the new cam used (the one with $4 \mathrm{~mm}$ eccentricity), the lubrication regime is still mixed but closer to the complete one than in the tests of the second group. In fact, the maximum value of the friction coefficient occurs between $200^{\circ}$ and $250^{\circ}$. This corresponds to $45^{\circ}$ after the maximum lift during the phase of increasing entraining speed when the squeeze effect narrows the film thickness. A similar effect is present in the previous test with the worn cam against the glass disc (Figure 20), but the highest value occurs at $90^{\circ}$ in correspondence of the worn zone.

More difficult is the interpretation of the lower friction coefficient between $0^{\circ}$ and $45^{\circ}$ and between $315^{\circ}$ and $360^{\circ}$ at $100 \mathrm{rpm}$ compared to 300 and $500 \mathrm{rpm}$. This could be related to the combination of several effects, as the one of the lower load (see Figure 11) and the different shift between film thickness and entraining speed variations under transient 
conditions at different frequencies, as reported in [14], but also to the sensitivity of the load cells measuring the friction components.

Other quantities can be easily evaluated from the measured ones. The total torque is due to friction and to the normal contact force. Friction effects are obviously always against the motion, but, as already mentioned at the end of paragraph 5.1, the normal force produces a positive moment for one half of the cam's rotation. In the sample diagram of Fig. 22 the moments due to friction and to the normal force are shown, as well as the total moment. The power losses for the cases of Fig. 21 is shown in Fig. 23.

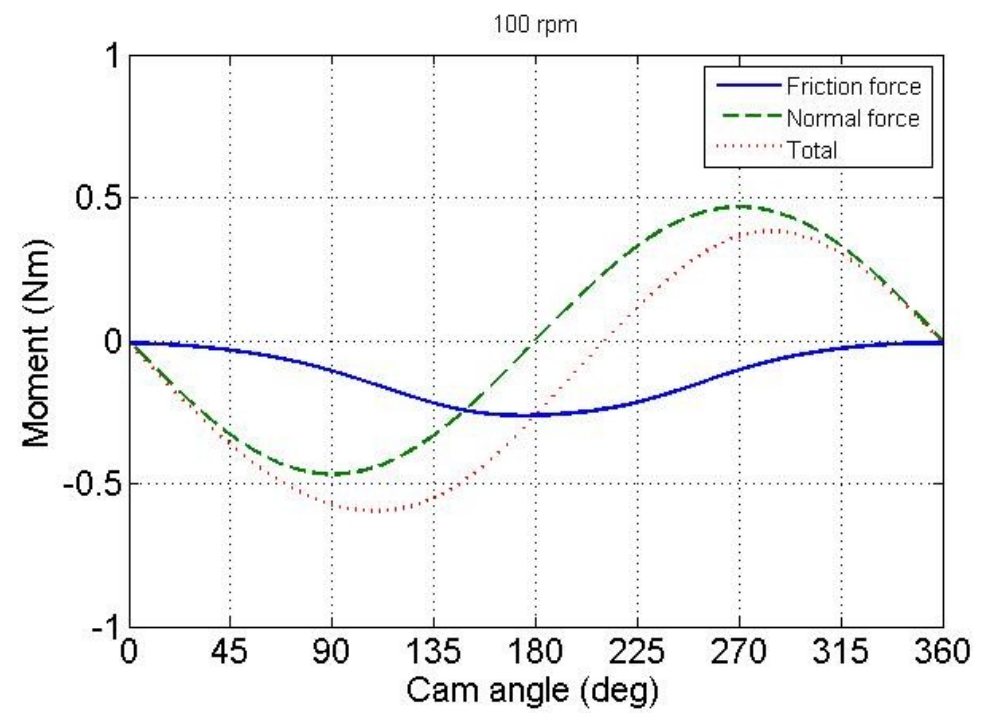

Figure 22 Torque components due to the tangential (friction) and normal contact forces and total torque versus cam rotation angle (third group of tests, $100 \mathrm{rpm}$ ).

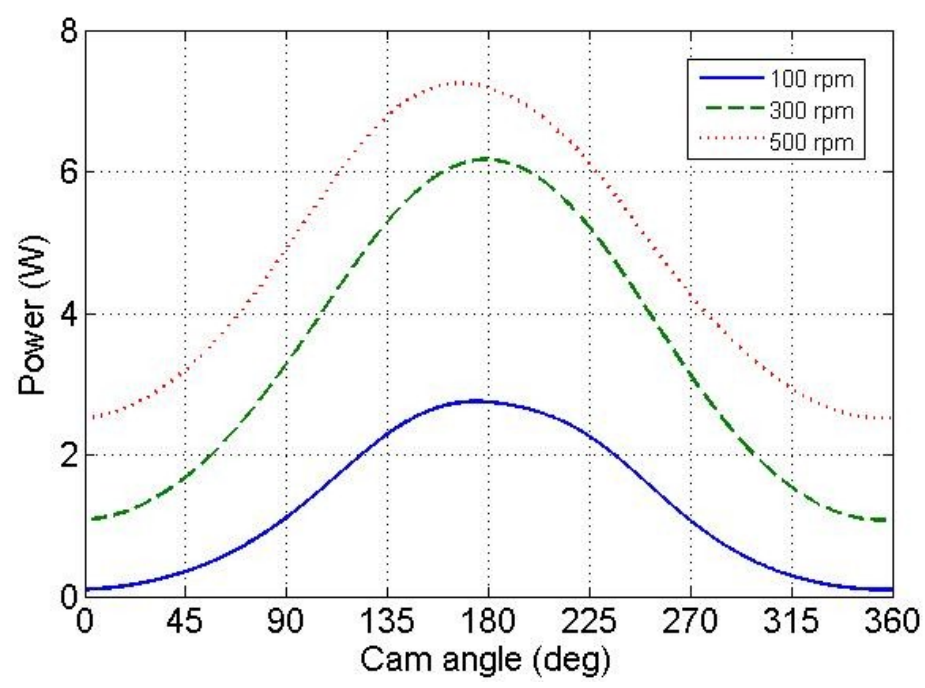

Figure 23 Friction power versus cam rotation angle in the third group of tests for three speed values.

A detailed evaluation of the film thickness from interference images has not been made in the present study, which is more addressed to test the capabilities of the experimental rig. On the other hand, the irregular roughness and geometrical shape of the cam as well as the presence of wear scratches make the detailed evaluation of the film thickness not very significant in this case. However, the results obtained indicate that with the test rig it is possible to record images under different test conditions. The oil film thickness and formation between cam and follower and their changes with the working condition will be investigated in detail in future works.

\section{Conclusions}

The capabilities of a new test rig for cam-follower lubricated pairs have been tested regarding with force and film thickness measurements with circular eccentric cams. Some first methodologies for numerical data elaboration have been identified. Sample results of normal and friction force and friction coefficient, as well as interference images 
usable for film thickness estimation are presented.

The use of circular eccentric cams with spherical surfaces has some advantages, as lower transient effects, the possibility to quite easily simulate the kinematic and dynamic behaviour and the absence of the contact alignment problems typical of cams with cylindrical surfaces. However, there are also some drawbacks, as the higher Hertzian pressure and higher and more localised wear. Non-uniformity in roundness and roughness were also evidenced during performing of the experiments.

Despite the above limitations, the obtained results furnish encouraging indications about the capabilities of the experimental apparatus. All force components have been measured with a sufficient data acquisition frequency under the different lubrication regimes ranging from boundary to complete. Optical interference images have been recorded correctly at the desired frequency. The contemporary measurements of all force components and film thickness allow a deep investigation of the phenomena occurring inside the lubricated contact under transient conditions.

The effects of the rotational speed, the preload, the oil viscosity, the cam eccentricity, the material and the surface roughness on oil film thickness, friction and wear can be studied in detail with the test rig.

\section{Acknowledgements}

Authors would like to thank Eng. Alessandro Paglini for the data manipulation and plots; Eng. Giacomo Bassi for the setting of camera apparatus and interferometric images; Eng. Dagoberto Vela for the test rig design, SKF for providing the rolling bearings of the test rig and Mollificio F.lli Ballotta for providing the calibrated springs.

\section{References}

[1] Vela, D., Ciulli, E., Piccigallo, B., Fazzolari, F., "Investigation on cam-follower lubricated contacts", Proc. IMechE, Part J: J. Engineering Tribology, 2011, 225(J6), 379-392.

[2] Dowson, D., Harrison, P. and Taylor, C.M., "The lubrication of automotive cams and followers", in Proc. 12th Leeds-Lyon Symposium on Tribology, Butterworths, Oxford, 305-322 (1986).

[3] Bair, S., Griffioen, J.A. and Winer, W.O., "The tribological behavior of an automotive cam and flat lifter system", Transaction of the ASME, Journal of Tribology, 108, 478-486 (1986).

[4] Soejima, M., Ejima, Y., Wakuri, Y. and Kitahara, T., "Improvement of lubrication for cam and follower", Tribology Transactions, 42, 4, 755-762 (1999).

[5] Gangopadhyay, A., Soltis, E. and Johnson, M.D., "Valvetrain friction and wear: influence of surface engineering and lubricants", Proc. Instn. Mech. Engrs., Journal of Engineering Tribology, 218, 147-156 (2004).

[6] Kano, M., "Super low friction of DLC applied to engine cam follower lubricated with ester-containing oil", Tribology International, 39, 1682-1685 (2006).

[7] Soejima, M. and Hamatake, T., "Tribology of Cam and Follower for Direct-type Valve Train", Proceedings of the 5th World Tribology Congress WTC2013, 8-13 September 2013, Torino, Italy, ISBN 978-88-90818509, p. 1-4.

[8] Priest, M. and Mufti,R.A., "Effect of cylinder pressure on engine valve-train friction under motored and fired conditions", Proc. IMechE, Part J: J. Engineering Tribology, 2012, 226(4), 306-314.

[9] Hamilton, G.M., "The hydrodynamic of a cam follower", Tribology International, 13, 113-119 (1980).

[10] van Leeuwen, H., Meijer, H. and Schouten, M., "Elastohydrodynamic film thickness and temperature measurements in dynamically loaded concentrated contacts: eccentric cam-flat follower", in Proc. 13th Leeds-Lyon Symposium on Tribology, Elsevier, Amsterdam, 611-625 (1987).

[11] Dowson, D., Harrison, P., Taylor, C.M. and Zhu, G., "Experimental observation of lubricant film state between a cam and bucket follower using the electrical resistivity technique", in Proc. Japan International Tribology Conference, Nagoya, Japan, 119-124 (1990).

[12] Bassani, R., Ciulli, E. and Forte, P., "Experiences of elastohydrodynamics on spheres and cams", in Proc. X AIMETA General Congress, Pisa 2-5 October 1990, Edizioni ETS, Pisa, Vol. 2, 427-432 (1990) (in Italian).

[13] Bassani, R., Ciulli, E., Carli, M. and Stadler, K., "Experimental investigation of Transient and Thermal Effects on Lubricated non-conformal Contacts", Tribotest, 13, 183-194 (2007).

[14] Ciulli, E., Draexl, T. and Stadler, K., "Film Thickness Analysis for EHL Contacts under Steady-State and Transient Conditions by Automatic Digital Image Processing", Advances in Tribology, Vol. 2008, Article ID 25187, 16 pages, 2008. doi:10.1155/2008/25187, http://www.hindawi.com/journals/at/volume-2008/, ISSN: 1687-5915, e-ISSN: 16875923, doi:10.1155/AT (2008).

[15] Ciulli, E., "Non-steady state non-conformal contacts: friction and film thickness studies", Meccanica, 44, 409-425 (2009). 
[16] Vela, D., Fazzolari, F., Bassani, R., Ciulli, E., "Set-up of a test rig for lubricated cam simulation", Proceedings of ECOTRIB 2011 - 3rd European Conference on Tribology, Vienna, Austria, June 7-9, 2011, The Austrian Tribology Society, Vienna, ISBN 978-3-901657-38-2, Vol.2, 545-550 (2011).

[17] Fazzolari, F., Ciulli, E., Vela, D., "A novel instrumentation for contact force measurement in cam-follower pairs", Proceedings of the $18^{\text {th }}$ International Colloquium Tribology, Industrial and Automotive Lubrication, 10-12 January 2012, Esslingen, Germany, 10 p., Technische Akademie Esslingen, ISBN 3-924813-97-3.

[18] Vela, D., Fazzolari, F., Ciulli, E., "Dynamic aspects of a new experimental apparatus for tribological investigations on cam-follower pairs", 13th World Congress in Mechanism and Machine Science, IFToMM 2011, 19-25 June, 2011, Guanajuato, México, on Flash pen, 9p. 